\title{
Flavor evaluative conditioning and contingency awareness
}

\author{
Susan G. Wardle, Chris J. Mitchell, and Peter F. Lovibond \\ University of New South Wales, Sydney, New South Wales, Australia
}

\begin{abstract}
The relationship between flavor evaluative conditioning and contingency awareness was examined in two experiments using flavored drinks. In Experiment 1, one flavor was always paired with sugar and the other with bitter tween ( polysorbate20) during conditioning. In a subsequent test phase, participants tasted the two flavors, and their evaluative ratings indicated an overall preference for the sugar-paired flavor. Moreover, participants were generally able to report which flavor had been paired with sugar and which with tween. This finding was replicated and confirmed in Experiment 2A. Furthermore, in both experiments, evaluative conditioning was seen only in those participants who were aware of the contingencies. Experiment $2 \mathrm{~B}$ demonstrated that evaluative conditioning does not occur to colors, although participants are contingency aware. The differences between the present findings and prior studies, in which apparently unaware flavor conditioning has been found, are discussed.
\end{abstract}

Evaluative conditioning occurs when a neutral stimulus acquires the affective valence of another stimulus with which it is paired (see De Houwer, Thomas, \& Baeyens, 2001 , for a review). In a typical procedure, the two stimuli are presented simultaneously and, following several repetitions, the conditioned stimulus (CS) is observed to acquire the affective valence of the unconditioned stimulus (US). One of the most actively debated issues in the evaluative conditioning literature is the relationship between evaluative conditioning and contingency awareness (De Houwer, 2001; Field, 2000; Fulcher \& Hammerl, 2001b; Hammerl, 2000; Lovibond \& Shanks, 2002; Shanks \& St. John, 1994). Although there is strong evidence that most examples of classical conditioning in humans require corresponding awareness of the stimulus contingencies (Lovibond \& Shanks, 2002), evaluative conditioning is often reported to occur only in the absence of awareness (Baeyens, Eelen, \& Van den Bergh, 1990; Fulcher \& Hammerl, 2001a; Levey \& Martin, 1975; Walther \& Nagengast, 2006). This feature, along with resistance to extinction (Baeyens, Crombez, Van den Bergh, \& Eelen, 1988; Vansteenwegen, Francken, Vervliet, De Clercq, \& Eelen, 2006) is sometimes used to distinguish evaluative conditioning from other forms of classical conditioning. However, the claim remains controversial and has been challenged by other researchers (Davey, 1994; Field \& Davey, 1999).

Lovibond and Shanks (2002) concluded that there is no substantial evidence in the evaluative conditioning literature that contradicts the view that conditioning occurs only in the presence of awareness. They emphasize that, in general, studies reporting conditioning without aware- ness use insensitive measures of participants' contingency knowledge. However, Lovibond and Shanks identified experiments which use flavors as the conditioned stimuli as providing some suggestive evidence for unaware evaluative conditioning.

Flavor conditioning is a variant of the evaluative conditioning procedure that has received surprisingly little attention. In the original demonstration (Zellner, Rozin, Aron, \& Kulish, 1983), participants drank a series of flavored teas. During conditioning, one of the tea flavors (CSpos) was always mixed with sugar, and the other was presented unsweetened (CSneg). Participants then preferred the tea flavor that had been paired with sugar during conditioning, even when it was presented in the absence of the US (i.e., unsweetened) on test.

Baeyens, Eelen, Van den Bergh, and Crombez (1990) were the first to explore the relationship between conditioning and awareness with flavors. The CSs were either fruit flavors or colors. Sugar was used as the positive US and tween (polysorbate 20, which has an unpleasant bitter taste) was the negative US. Baeyens, Eelen, et al. reported an interesting dissociation between conditioning and awareness. Participants exhibited flavor-flavor evaluative conditioning and rated the flavor that had been paired with sugar as more liked than the flavor that had been paired with tween. However, they showed no awareness of the CS-US contingencies. In contrast, participants did not show any evidence of color-flavor evaluative conditioning, yet they could reliably identify which colors had been paired with sugar and which with tween earlier in the experiment. The demonstration of unaware flavor conditioning has some weight because the awareness test was

C. J. Mitchell, chris.mitchell@unsw.edu.au 
sensitive enough to detect awareness of the color-flavor contingencies. Evaluative conditioning occurred with flavor CSs, yet participants could not accurately report the stimulus contingencies.

In the Baeyens, Eelen, et al.'s (1990) measure of awareness, participants were explicitly informed in the test phase that some of them had received sugar in their drinks, others tween, and others just the fruit flavors. On a response sheet, participants were required to indicate what flavor (or color) of CS the sugar (or tween) US was presented with most of the time, and how certain they were of their answer. The flavor and color stimuli were placed in front of participants, and they were permitted to smell and taste the test stimuli as many times as they wished before answering the questions. The names of the test stimuli were written underneath each cup; once participants had made their selections on the basis of the sensory characteristics of the stimulus, they confirmed the name of the CS by consulting the label in order to answer the questions.

A weakness of this awareness measure is that the awareness test procedure was not identical to the test of evaluative conditioning. During the evaluative test, and also in the conditioning phase, participants tasted the drinks in a precise timed sequence, whereas in the awareness test they could taste each drink as little or as often as they wished. Lovibond and Shanks (2002) stressed that the conditions experienced in the learning and testing phases should be as similar as possible in order to increase the sensitivity of the test. One might, of course, argue that Baeyens, Eelen, et al.'s (1990) test would be more sensitive because multiple tastings were allowed. However, the possibility that this test procedure might have confused participants is enough to cast doubt on the claim that the participants were appropriately classified as aware and unaware in that experiment; what is needed is a contingency awareness test as similar as possible to the test of evaluative conditioning.

An additional concern with the Baeyens, Eelen, et al. (1990) measure is that the order in which the evaluative and awareness tests were completed was not counterbalanced. As the awareness test was always completed second, it is possible that the evaluative ratings were affecting the awareness measure or that the lack of knowledge of flavor CS contingencies was simply due to forgetting. However, in spite of these shortcomings the test was still sensitive enough to detect participants' knowledge of the color contingencies, and this is the main strength of Baeyens, Eelen, et al.'s results.

Dickinson and Brown (2007) have provided the only replication of unaware flavor conditioning that we are aware of; but see Stevenson, Boakes, and Prescott (1998) for related studies. Dickinson and Brown's focus was not contingency awareness but blocking of flavor evaluative conditioning by color-taste pairings, for which they found no evidence. Nevertheless, their evidence for unaware flavor conditioning is the best in the literature. Dickinson and Brown used a somewhat improved experimental design partially based on parametric experiments by Baeyens, Crombez, Hendrickx, and Eelen (1995) and suggestions by Lovibond and Shanks (2002) on what constitutes an ap- propriate and sensitive measure of contingency awareness. Dickinson and Brown's awareness measure was superior to that of Baeyens, Eelen, et al. (1990), in that Dickinson and Brown used a timed tasting procedure identical to that used in conditioning, and the same continuous rating scales for both the evaluative and the contingency tests. For each US (sugar and tween), participants were asked to rate their level of certainty that it had been presented with each flavor. However, as in Baeyens, Eelen, et al.'s (1990) study, the order in which the evaluative and contingency tests were completed was not counterbalanced. In agreement with Baeyens, Eelen, et al. (1990), Dickinson and Brown reported evaluative conditioning with flavors despite an absence of contingency awareness. Although the pattern of responding that emerged (as averaged across participants) was in the direction expected if participants were aware of the CS-US contingencies, the mean ratings were close to zero. Dickinson and Brown concluded that their participants were not aware.

It is remarkable that there are only a handful of studies that use the flavor-flavor paradigm, because preliminary work indicates that it is one of the most promising avenues for investigating the relationship between evaluative conditioning and awareness. The two studies published thus far have both reported that conditioning occurred in the absence of any explicit knowledge of the stimulus contingencies. Although this is an intriguing result, it is still possible that the tests of awareness have not yet been sensitive enough to detect knowledge of the stimulus contingencies. To make a case for unaware conditioning, all that is required is an insensitive measure of awareness. For example, Dickinson and Brown's (2007) contingency test was always completed second, so it is possible that this delay interfered with participants' recollection of the CS-US contingencies. It is, therefore, worthwhile to attempt to replicate the flavor conditioning result.

\section{EXPERIMENT 1}

Experiment 1 was designed to replicate the basic evaluative conditioning effect reported by Baeyens, Eelen, et al. (1990) and Dickinson and Brown (2007), and to further investigate whether it is possible to detect any level of contingency awareness at all in flavor evaluative conditioning. We used a within-subjects design with two flavors as CSs. For each participant one flavor was always paired with sugar during conditioning and the other with tween. As in Baeyens, Eelen, et al., the flavor CSs were presented in a variety of artificial colors in the conditioning phase. The design of our awareness test was similar to Dickinson and Brown. Lovibond and Shanks (2002) argued that the most sensitive post-conditioning measure of awareness is a recognition rather than a recall test, with a continuous rating scale that is administered immediately after conditioning. Following Dickinson and Brown, we used an identical continuous scale for both the evaluative and contingency measures in order to equate the sensitivity of the tests. In the test phase, participants tasted the CS flavor and completed both the evaluative and contingency rating scales for that flavor before proceeding to the next 
flavor. In addition, the timed tasting procedure used in the conditioning and test phases was identical.

The main differences between our measure of awareness and Dickinson and Brown's (2007) were (1) the question format and (2) the way in which we counterbalanced the order of completion of the evaluative and awareness measures. In Dickinson and Brown, participants were asked to identify which CS went with the USs (sugar and tween). There were separate answer sheets for the sugar and tween contingencies with the question "Which, if any, of the following flavors was SUGAR (or TWEEN) always presented with?" and a rating scale for each of the four CS flavors. In our test, the question was reversed. For each flavor of CS (there were only two in our experiment), participants were asked to rate their level of certainty that it had been paired with each US (sugar and tween). We assumed that this would be less confusing for participants, because on each trial of the test phase participants tasted a particular flavor CS; thus, the focus was on the CS instead of on the US. For each CS there was one answer sheet which contained both the sugar and tween questions. We also adjusted the question wording used by Dickinson and Brown to make it more natural - for example, "Was this flavor mixed with a SWEET (or BITTER) taste most of the time in this experiment?" instead of asking what CS flavor was presented with sugar or tween.

\section{Method}

Participants. The participants were 48 undergraduate students at the University of New South Wales (12 males, 36 females, mean age 19) who participated voluntarily for partial course credit. They were not told the purpose of the experiment and had no food allergies.

Stimuli. Two Queen concentrate flavoring essences (orange and vanilla) were used as the CSs. The flavors were selected on the basis of an independent pilot study in which participants tasted and evaluated several flavors. Flavor solutions were at a concentration of $20 \mathrm{ml} / 1$ (orange) and $40 \mathrm{ml} / 1$ (vanilla). Sugar (40 g/l water) and tween (polysorbate 20) at the concentration of $2 \mathrm{ml} / 1$ water (as used in Baeyens et al., 1990) were the positive and negative USs, respectively. Samples of flavor solution $(5 \mathrm{ml})$ were presented for tasting at room temperature in 30- $\mathrm{ml}$ transparent plastic cups. Artificial food coloring (Queen) was used to color the flavor samples. Each flavor CS was presented a total of six times during conditioning; once each in red, yellow, green, blue, pink, and colorless.

Design. The experiment used a within-subjects design. For each participant, one flavor was always paired with sugar (CSpos) and the other with tween (CSneg) during conditioning. The two dependent variables were participants' evaluative and contingency ratings for the CSpos and CSneg. The allocation of flavors to the roles of CSpos and CSneg was counterbalanced, as was the order of the evaluative and contingency tests and which of the two flavors was presented first in the test phase. Participants were randomly assigned to one of the resulting eight $(2 \times 2 \times 2)$ counterbalancing conditions.

Procedure. Participants were tested individually in a small testing room. At the start of the half-hour session, the experimenter emphasized that although some of the drinks might taste unpleasant, they were all completely harmless. The conditioning phase consisted of 12 samples (6 CSpos and 6 CSneg). Participants were informed that in the first phase of the experiment they would be required to taste the 12 samples. They were instructed to focus on the taste of each sample and to try to remember it because it would be relevant later in the experiment. Each conditioning trial was $20 \mathrm{sec}$ long, with a $20-\mathrm{sec}$ intertrial interval. The tasting procedure consisted of a series of 5-sec stages, in the following order: In the first stage, each partici- pant picked up the sample and looked at it; for the next $5 \mathrm{sec}$, the participant smelled the sample; then, during the following $5 \mathrm{sec}$, tasted it and, as instructed, swirled the entire sample around the tongue. In the final 5-sec phase, the particpant expectorated the drink sample into a cup and, to clear the palate, took a bite of a plain water cracker. There was a $20-\mathrm{sec}$ waiting phase in between each trial.

The experimenter explained the tasting procedure in detail and for reference the instructions for each stage were also posted on the wall in front of the participant. The experimenter was seated at a computer and used custom-written software to monitor the timing of each stage of the tasting procedure. Each participant was guided through the tasting with verbal instructions for each stage; "look," "smell," "taste," "spit and cracker." Participants tasted the samples in a unique semirandom order generated by a computer program; the sole restriction was that the same flavor could not occur more than twice consecutively.

In the test phase, the flavor CSs were presented colorless and in the absence of the US (i.e., in plain water). There were two test trials; one for each CS. The participant tasted one CS and then filled out both the evaluative and the contingency ratings for that flavor. The tasting procedure was identical to that used in the conditioning phase, except that participants were required to answer the questions in the waiting period. They were given as long as they needed to respond, but it was emphasized that participants should give their first, immediate, and spontaneous response to the questions, and should avoid thinking about them too much.

In the evaluative test, participants first tasted the sample and then answered the question "How much did you like this flavor?" on a scale from -10 (very disliked) to 0 (neutral) to +10 (very liked). In the test of contingency awareness, participants tasted the sample and then answered the following two questions: "Was this flavor mixed with a SWEET taste most of the time in this experiment?" and "Was this flavor mixed with a BITTER taste most of the time in this experiment?" on a scale from -10 (definitely wasn't) to 0 (don't know) to +10 (definitely was).

\section{Results and Discussion}

To analyze the data from the evaluative and contingency ratings, a set of contrasts was tested using a multivariate model (O'Brien \& Kaiser, 1985), with the three counterbalancing variables as between-subjects factors and the CSpos and CSneg as repeated measures. This approach was used for all of the following analyses, and also in Experiments $2 \mathrm{~A}$ and $2 \mathrm{~B}$.

Counterbalancing. None of the between-subjects counterbalancing conditions of flavor role (CSpos or CSneg), order of test completion, or order of flavor presentation on test interacted with the within-subjects manipulation on either dependent variable [greatest $F(1,40)=$ $1.507, p>.05]$, so the data from all participants were combined for analysis.

Evaluative ratings. Figure 1A presents the mean evaluative ratings for the CSpos and CSneg. The ratings indicate that both CSs were perceived to be mildly unpleasant. However, as expected, participants rated the CSpos as more liked than the CSneg $[F(1,40)=6.797, p<.05]$. Therefore, the evaluative conditioning procedure was successful; participants liked the flavor that had been paired previously with sugar (CSpos) more than the flavor that had been paired with tween (CSneg).

Contingency awareness. Figure 1B shows the results from the awareness test. Positive ratings indicate participants' level of certainty that a particular CS was paired with a particular US (sweet or bitter). Conversely, negative ratings indicate certainty that a CS was not paired with 

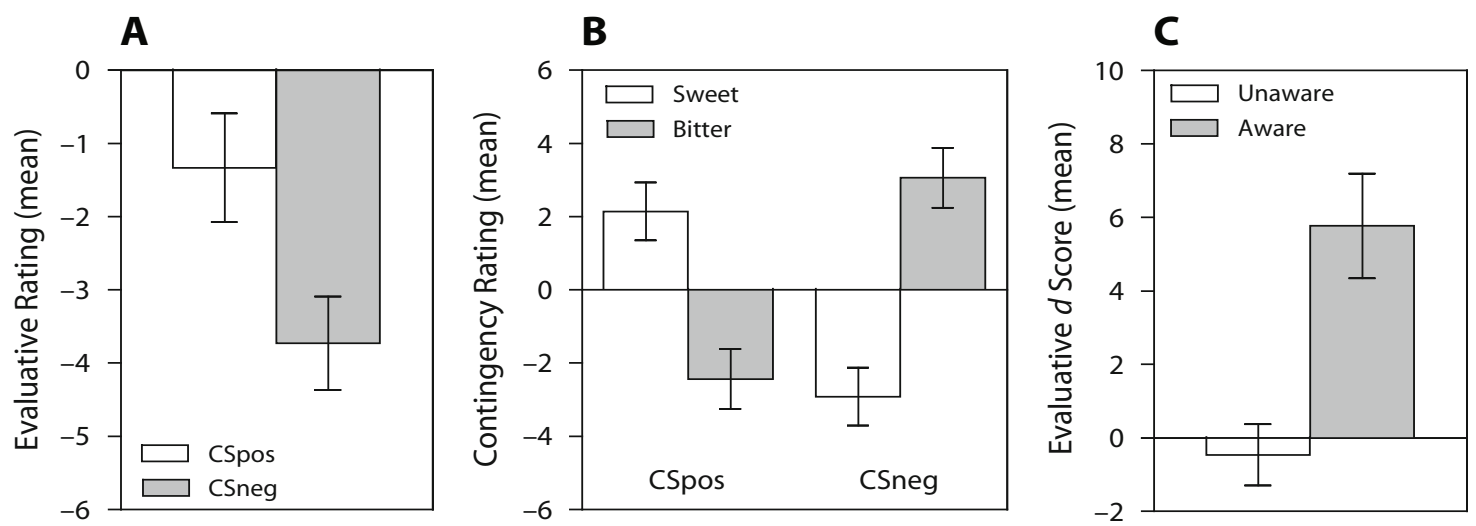

Figure 1. Data from Experiment 1. (A) Mean evaluative ratings for the CSpos and CSneg. (B) Mean contingency ratings for the CSpos and CSneg. Ratings indicate participants' level of confidence that the CS was paired with a sweet or a bitter taste in the first phase of the experiment. (C) Amount of evaluative conditioning in aware and unaware participants. Error bars on all graphs indicate standard errors of the means.

a particular taste. Therefore, if participants are aware of the CS-US contingencies they should rate the CSpos as more likely to have been paired with a sweet than a bitter taste, and show the inverse response pattern for the CSneg. The level of contingency knowledge for each CS is thus assessed by comparing the contingency ratings for sweet and bitter awarded to that CS.

There was a significant interaction between CS type (CSpos or CSneg) and participants' ratings for sweet and bitter $[F(1,40)=20.641, p<.001]$. This reflects the fact that for the CSpos, participants gave a positive contingency rating for sweet and a negative rating for bitter. In contrast, for the CSneg, participants rated sweet negatively and bitter positively. Therefore, the results suggest that on average, participants were aware of the CS-US contingencies.

\section{Relationship Between Evaluative Conditioning and Awareness}

Participants were classified as aware or unaware in order to examine the relationship between awareness and evaluative conditioning. Participants were considered to be aware of a CS-US contingency if, for a given CS, they rated the paired US as more likely to have been paired with that CS than the unpaired US. For example, to be considered aware of the sugar-paired contingency, a participant would have to give a higher rating for that $\mathrm{CS}$ on the sweet scale than on the bitter scale. As there were two CSs, chance responding was determined to be answering one of the contingency questions correctly. Thus, participants who scored 0 or 1 were classified as unaware $(n=26)$, and only those participants who answered both contingency questions correctly were classified as aware $(n=22)$.

As shown in Figure 1C, only the aware group displayed evaluative conditioning, measured by the difference between their ratings for the CSpos and CSneg. The difference score for the aware group was significantly greater than zero $[t(21)=4.047, p<.01]$, confirming that their evaluative ratings for the CSpos were significantly higher than were those for the CSneg. However, the score for the unaware group was not different from zero $(t<1)$, indi- cating an absence of evaluative conditioning. Moreover, the difference between the evaluative difference scores for the aware and unaware groups was significant $[t(46)=$ $3.909, p<.01]$.

Experiment 1 successfully replicated the flavor evaluative conditioning effect reported by Baeyens, Eelen, et al. (1990) and Baeyens et al. (1995) and Dickinson and Brown (2007). However, unlike participants in those previous studies, a large number of participants were aware of the CS-US contingencies. When participants were divided into groups on the basis of their level of awareness, it became clear that only aware participants demonstrated an evaluative conditioning effect. This is a striking result; it contradicts the reports of evaluative conditioning in the absence of awareness of Baeyens, Eelen, et al. (1990) and of Dickinson and Brown. However, there is an alternative explanation for the results that needs to be eliminated. A concern with this experiment is that one of the flavors (vanilla) had a slight yellowish color in the concentration used. This means that participants may have used the color of the samples rather than the flavor to remember which tasted bitter and which was sweet; if so, they could have answered the contingency questions accurately without having any knowledge of the flavor contingencies. Although this is unlikely because this subtle color should have been masked by the colors added during training, the problem will be addressed in Experiment 2 by using colorless flavors.

An additional methodological issue with Experiment 1 is that the counterbalancing of the evaluative and contingency questions may not have been effective because they were printed on the same side of the answer sheet, and participants tended to read through all of the questions on the first test trial before responding. In Experiment 2, the evaluative and contingency questions were printed on separate pages of the answer booklet to ensure effective counterbalancing.

\section{EXPERIMENT 2A}

The aim of this experiment was to replicate and confirm the results of Experiment 1. The main shortcoming of Ex- 
periment 1 was that the vanilla flavor had a slight yellowish color that may have aided participants in answering the questions on the contingency test. Consequently, two colorless flavors were used in Experiment 2A.

\section{Method \\ Participants. Thirty-two undergraduate students from the Uni- versity of New South Wales (17 males, 15 females) with a mean age of 18.9 years and no food allergies participated in Experiment $2 \mathrm{~A}$ for partial course credit. \\ Stimuli. The stimuli were the same as those for Experiment 1 , except that the flavors used as CSs were orange and coconut. Both of these flavors are colorless at the concentration used $(20 \mathrm{ml} / 1)$. In addition, in this experiment only two colors (blue and yellow) were used in the conditioning phase. Each CS was presented in each color three times. On test, one CS was presented in pink and the other in green.}

Design. The experiment was a within-subjects design; the two dependent variables were participants' evaluative and contingency ratings for the CSs. The assignment of orange and coconut as CSpos and CSneg was counterbalanced. In addition, also completely counterbalanced were the order in which the evaluative and contingency tests were administered, the CS that was tested first, and the color of each CS in the test phase. Participants were randomly assigned to one of the resulting $16(2 \times 2 \times 2 \times 2)$ counterbalancing conditions.

Procedure. The procedure was the same as in Experiment 1, except in the following respects. The evaluative and contingency questions were printed on separate pages of the answer booklet. In addition, there were separate test trials for the evaluative and contingency questions. This created four test samples, because each of the two CSs was presented twice. The first measure was completed for both CSs before the second measure was administered. To encourage participants to give their first and immediate response on the evaluative and contingency ratings, they were given only $40 \mathrm{sec}$ to answer the questions on each test. The wording of the question on the evaluative test was altered slightly from that used in Experiment 1, with the word "drink" replacing "flavor" (e.g., "How much do you like this drink?").

\section{Results and Discussion}

Counterbalancing. None of the between-subjects counterbalancing conditions interacted with the withinsubjects manipulation on either dependent variable [greatest $F(1,16)=1.845, p>.05]$, so the data from all participants were combined for analysis. There was a significant main effect for question order $[F(1,16)=11.820, p<$ $.01]$. However, this appeared to be due to variations in the level of participants' ratings on the contingency test that did not affect the pattern of the data and did not interact with the comparisons of interest.

Evaluative ratings. As displayed in Figure 2A, in the test phase, the two flavor CSs both received negative ratings, indicating that they were perceived by participants as mildly unpleasant. However, as in Experiment 1, participants rated the flavor that had been paired with sugar (CSpos) as more liked than the flavor that had been paired with tween (CSneg). This difference was significant $[F(1,16)=7.202, p<.05]$. Therefore, in the conditioning phase, pairing a flavor with sugar resulted in a more positive evaluation of the flavor when it was later presented on its own on test than did pairing it with tween.

Contingency awareness. As in Experiment 1, the level of contingency knowledge for each CS was assessed by comparing participants' ratings of the likelihood that it was paired with a sweet and a bitter taste during conditioning. Figure 2B reveals that for the CSpos, participants rated the sweet contingency positively and the bitter contingency negatively. On the other hand, for the CSneg, the mean ratings were negative for the sweet contingency and positive for the bitter contingency. The interaction between CS type (CSpos or CSneg) and the sweet and bitter ratings was significant $[F(1,16)=19.049, p<.001]$. Thus, participants were aware of the CS-US contingencies.

\section{Relationship Between Evaluative Conditioning and Awareness}

Participants were again divided into unaware $(n=15)$ and aware $(n=17)$ groups, using the criteria described in Experiment 1. The results are presented in Figure 2C. Only the aware group displayed evaluative conditioning, as measured by the difference between their ratings for the CSpos and CSneg. This observation was confirmed by a test of whether the aware group's evaluative difference score was significantly above zero $[t(16)=4.343, p<$ $.01]$. The difference score for the unaware group did not differ significantly from zero $(t<1)$, confirming an absence of evaluative conditioning. Furthermore, the aware group had a significantly higher evaluative conditioning score than the unaware group $[t(30)=3.314, p<.01]$. Therefore, as in Experiment 1, only participants who were aware of the stimulus contingencies exhibited an evaluative conditioning effect.

These findings are inconsistent with those of Baeyens, Eelen, et al. (1990) and Dickinson and Brown (2007). We shall return to this issue in the General Discussion; first, though, we shall examine Baeyens, Eelen, et al.'s other interesting finding, that evaluative conditioning was not observed using color CSs despite awareness of the CS-US contingencies. This result will have implications for the interpretation of Experiments 1 and 2A.

\section{EXPERIMENT 2B}

The positive relationship between evaluative conditioning and awareness in Experiments 1 and 2A suggests that this form of learning does not differ from other types of classical conditioning in humans (Lovibond \& Shanks, 2002). If evaluative conditioning is not a unique form of learning, it should presumably occur to any kind of CS. Thus, it is interesting that Baeyens, Eelen, et al. (1990) did not find evaluative conditioning to color CSs, although participants were aware of the stimulus contingencies. In the evaluative test in Baeyens, Eelen, et al., participants were asked to rate the colors on a scale of "very ugly" to "very pretty." It is possible that their failure to find evaluative conditioning is because participants' evaluation of a color following conditioning with tastes does not alter their perception of how visually attractive a color is, but instead brings to mind sweetness or bitterness. If this is the case, an evaluative test appropriate for color CSs needs to be administered.

In this experiment, we attempted to increase the sensitivity of the test for evaluative conditioning with color 

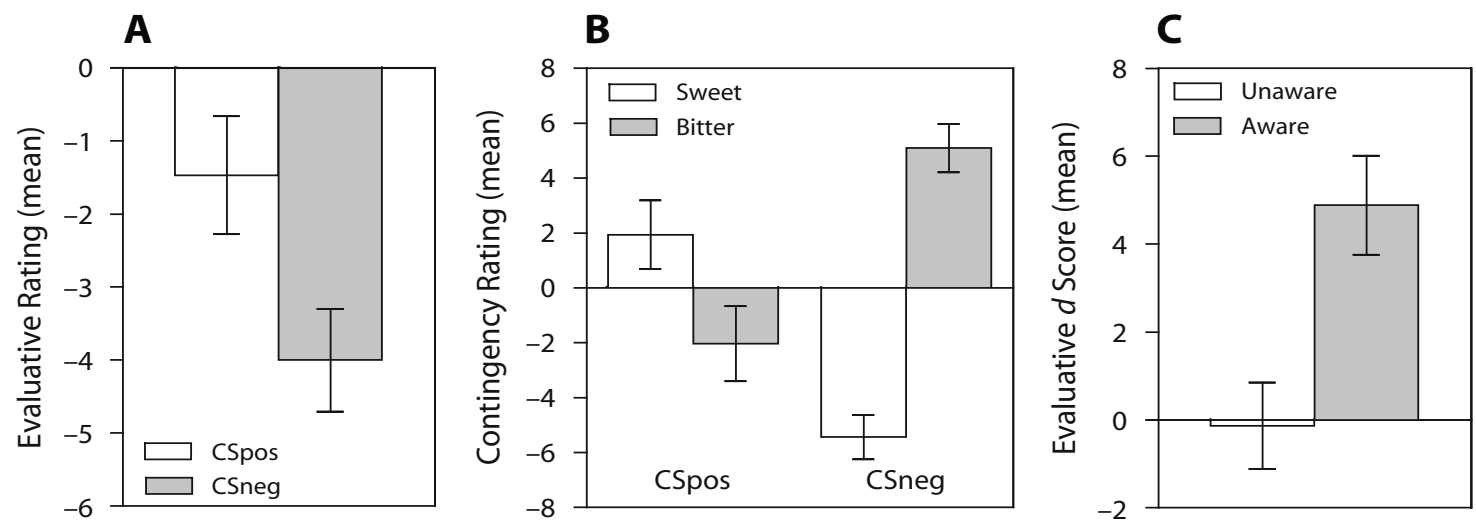

Figure 2. Data from Experiment 2A. (A) Mean evaluative ratings for the CSpos and CSneg. (B) Mean contingency ratings for the CSpos and CSneg. Ratings indicate participants' level of confidence that the CS was paired with a sweet or a bitter taste in the first phase of the experiment. (C) Amount of evaluative conditioning in aware and unaware participants. Error bars on all graphs indicate standard errors of the means.

CSs by presenting the colors in a novel flavor on test. The rationale is that if evaluative conditioning does cause a sensation of sweetness or bitterness to become associated with the CS, it should influence the evaluation of a new flavor presented with the CS. We would therefore expect that when participants evaluate the drinks, the novel flavor presented in the color conditioned with sugar will be preferred to the novel flavor presented in the color conditioned with tween. This manipulation also allowed us to ask a more general evaluative question with the same wording as in Experiment 2A ("How much do you like this drink?"). Apart from the fact that the tastes were contingent on the colors rather than on the flavors, all details were the same as in Experiment 2A, to allow for direct comparison.

\section{Method}

Participants. Thirty-two undergraduate students from the University of New South Wales (4 males, 28 females, mean age 19.6), with no food allergies, participated in Experiment 2B for partial course credit.

Stimuli. Pink and green food coloring served as the CSs and four Queen concentrate flavoring essences were used as distractors; lemon $(20 \mathrm{ml} / \mathrm{l})$, rosewater $(40 \mathrm{ml} / 1)$, orange $(20 \mathrm{ml} / 1)$, and coconut $(20 \mathrm{ml} / \mathrm{l})$. All flavors are colorless at the concentration used. In the conditioning phase, both colors were mixed three times with lemon and three times with rosewater, producing a total of 6 trials for each $\mathrm{CS}$. In the test phase, one color was mixed with orange and the other with coconut; the test drinks were, therefore, identical to those used in Experiment 2A.

Design. The experiment was a within-subjects design; the two dependent variables were participants' evaluative and contingency ratings for the CSs. The assignment of pink and green as CSpos and CSneg was counterbalanced. In addition, the order in which the evaluative and contingency tests were administered, the CS that was tested first, and the flavor of each CS in the test phase were also completely counterbalanced. Participants were randomly assigned to one of the resulting $16(2 \times 2 \times 2 \times 2)$ counterbalancing conditions.

Procedure. The procedure was the same as that used in Experiment $2 \mathrm{~A}$.

\section{Results and Discussion}

Counterbalancing. None of the between-subjects counterbalancing conditions interacted with the within- subjects manipulation on either dependent variable [greatest $F(1,16)=3.228, p>.05]$, so the data from all participants were combined for analysis.

Evaluative ratings. Figure $3 \mathrm{~A}$ confirms that participants perceived both test drinks as mildly unpleasant, as in Experiments 1 and 2A. However, in this experiment there was no significant difference between participants' evaluative ratings for the CSpos and their ratings for the CSneg $(F<1)$. It appears that pairing a color with sugar or tween did not alter the evaluation of a new flavor presented in that color in the test phase. Thus, in agreement with Baeyens, Eelen, et al. (1990), we found evaluative conditioning with flavor CSs in Experiments 1 and 2A, but not with color CSs in Experiment 2B. Presenting the colors in a novel flavor on test did not reveal any evaluative conditioning to the colors; thus, there was no evidence to support the suggestion that colors invoke an association of sweetness or bitterness when conditioned with tastes.

Contingency awareness. Contingency awareness was assessed as in Experiment 2A. The mean contingency ratings for the CSpos and CSneg are displayed in Figure 3B. Overall, for the CSpos, participants correctly rated the color as more likely to have been paired with a sweet than with a bitter taste. In addition, participants on average correctly rated the CSneg as more likely to have been paired with a bitter than with a sweet taste. There was a significant interaction between CS type (CSpos or CSneg) and the sweet and bitter ratings $[F(1,16)=5.593, p<.05]$. It thus appears that, on average, participants were aware of the CS contingencies.

\section{Relationship Between Evaluative Conditioning and Awareness}

Participants were divided into unaware $(n=17)$ and aware $(n=15)$ groups, using the criteria outlined in Experiment 1. The results are shown in Figure 3C. Neither the aware nor the unaware group had a difference score that was significantly different from zero (both $t \mathrm{~s}<1$ ). Moreover, there was no significant difference between the evaluative conditioning scores for the unaware and aware 

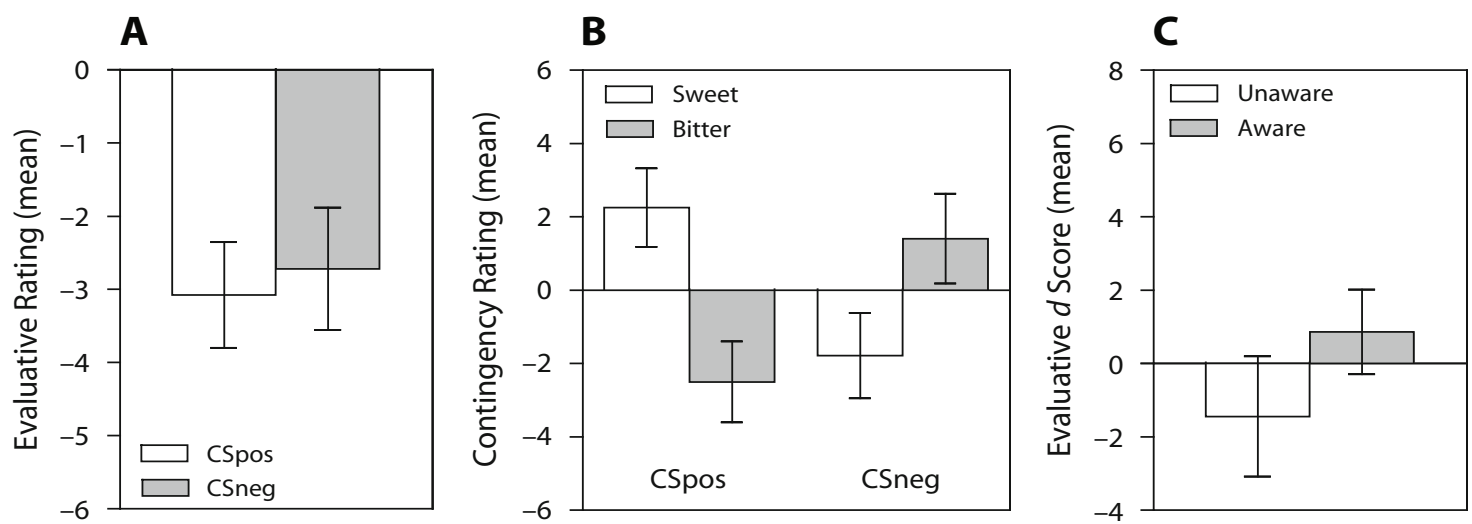

Figure 3. Data from Experiment 2B. (A) Mean evaluative ratings for the CSpos and CSneg. (B) Mean contingency ratings for the CSpos and CSneg. Ratings indicate participants' level of confidence that the CS was paired with a sweet or a bitter taste in the first phase of the experiment. (C) Amount of evaluative conditioning in aware and unaware participants. Error bars on all graphs indicate standard errors of the means.

groups $[t(30)=1.124, p>.05]$. Thus there was no evaluative conditioning to color CSs, regardless of whether or not participants were aware of the contingencies.

The procedure used in the present experiment was the same as that used in Experiment 2A, with one exception: Rather than conditioning flavor CSs and testing them in a novel color, we conditioned color CSs and tested them in a novel flavor. Although participants were aware of the CS-US (color-taste) contingencies, they showed no evaluative conditioning. This supports Baeyens, Eelen, et al.'s (1990) suggestion that there are important and interesting differences between colors and flavors in evaluative conditioning.

\section{GENERAL DISCUSSION}

This study reported three main findings. Firstly, we replicated the Baeyens, Eelen, et al. (1990) and Baeyens et al. (1995) flavor conditioning effect in two experiments. Two flavors, orange and vanilla in Experiment 1 and orange and coconut in Experiment 2A, served as CSs in a classical conditioning procedure. In the test phase, participants preferred the flavor that had been paired in training with sugar (CSpos) to the flavor that had been paired in training with tween (CSneg). Therefore, the conditioning procedure appears to have been successful in altering participants' relative liking for the flavor CSs.

Secondly, participants in all experiments reported awareness of the CS-US contingencies. In the test of awareness, participants tasted each CS flavor (or color) in plain water and rated the likelihood that it was mixed with a sweet (sugar) or bitter (tween) taste during the conditioning phase. Overall, participants were able to correctly identify the US with which each CS had been paired, and additionally, the US with which it had not been paired. Furthermore, Experiment 2B failed to obtain an evaluative conditioning effect with colors. In agreement with Baeyens, Eelen, et al. (1990), participants were aware of the contingencies yet did not display evaluative conditioning.
The final and most interesting result from the present study is that only aware participants in Experiments 1 and 2A exhibited an evaluative conditioning effect. Although previous studies have reported a similar relationship between awareness and evaluative conditioning using visual stimuli (Fulcher \& Cocks, 1997; Pleyers, Corneille, Luminet, \& Yzerbyt, 2007; Shimp, Stuart, \& Engle, 1991), the present study is the first to demonstrate a positive relationship between conditioning and awareness in flavor evaluative conditioning.

The fact that only aware participants displayed evaluative conditioning in this study is striking because it is inconsistent with the results of Baeyens, Eelen, et al. (1990) and Dickinson and Brown (2007), who reported evaluative conditioning in the absence of awareness of the CS-US contingencies. As previously discussed, the main weakness of the Baeyens, Eelen, et al. awareness measure is that it was considerably different from the conditioning phase, whereas the measure of evaluative conditioning was very similar. Moreover, it was always administered after the evaluative test, which may have reduced participants' ability to recall the contingencies. However, the measure of awareness used by Dickinson and Brown was very similar to that in the present study, so the conflicting results are particularly interesting. Dickinson and Brown's participants did not show a significant effect of contingency awareness on aggregate scores. It is important to note, though, that the mean responses on the contingency questions were in the direction that would indicate awareness.

There are several explanations why our procedure may have boosted the overall level of contingency awareness. One is that we altered the awareness measure slightly from Dickinson and Brown's (2007), in an effort to make it more sensitive. However, we concede that the changes to the question wording and format may seem too minor to account for the discrepancy in results. Another is that all participants in Dickinson and Brown were tested for awareness after the evaluative conditioning test, whereas the order of the tests was counterbalanced in the present 
article. Thus, the contingencies may have been more difficult to recall in their experiment. However, this explanation is undermined by the absence of any effect of test order in our experiments.

Perhaps the critical difference between the experiments lies in the training procedure, rather than at test. Thus, the instruction at the start of the conditioning phase to pay attention to the tastes and try to remember them may have encouraged participants to approach our experiment as a problem-solving task. This may have focused attention on the contingencies. Another possibility is that our experimental design was less complex. The two CS contingencies in our experiment would have been comparably easier for participants to identify than the four contingencies in Dickinson and Brown (2007) would have been. If this is the case, we would have to concede that Dickinson and Brown successfully demonstrated unaware evaluative conditioning and that to do so, it is necessary to use a complex experimental design. However, before we accept this conclusion, it is worth examining Dickinson and Brown's data more closely.

Dickinson and Brown's (2007) analysis of contingency awareness was on aggregate scores, and we thought it was possible that this might disguise some contingency awareness - either in a subset of participants or for a subset of individual CSs. Pleyers et al. (2007) have recently demonstrated that a single data set can produce opposing conclusions about the relationship between evaluative conditioning and awareness, depending on the level at which it is analyzed. Awareness of the relationship between a subset of CSs and the US with which they were paired can drive an overall evaluative conditioning effect, but this contingency awareness might be hidden when analyzed together with the awareness scores for other CSs. Dickinson and Brown kindly supplied us with their raw data so that we could conduct a more detailed analysis of the relationship between conditioning and awareness in their experiment.

We classified individual participants in Dickinson and Brown (2007) as aware or unaware, according to the criteria we used in our experiments. To be classified as aware of a contingency, participants were required to rate the paired taste (sugar or tween) as more likely than the unpaired taste to have been paired with that CS. As there were four contingencies, chance responding was considered to be scoring two out of four correct. Thus, we classified participants in Dickinson and Brown's study as aware if they scored correctly on at least three out of the four contingencies $(n=12)$, and unaware if they were correct for two or fewer contingencies $(n=20)$. Evaluative conditioning in Dickinson and Brown (2007) was measured by the difference between pretest and posttest evaluations of the flavor CSs. Thus, we calculated an overall measure of evaluative conditioning for each participant in their experiment by summing their difference scores across the four CSs. The difference scores for the tween CSs were reverse scored prior to this calculation, because negative scores indicated evaluative conditioning. Figure 4 presents the mean level of evaluative conditioning for the unaware and aware groups in Dickinson and Brown.
It is clear that the mean evaluative conditioning score was higher for aware than for unaware participants. The difference score for the aware group was significantly above zero $[t(11)=4.690, p<.01]$, confirming an evaluative conditioning effect. However, the score for the unaware group was not $[t(19)=1.875, p>.05]$. Moreover, the difference between the evaluative conditioning scores for the aware and unaware groups was significant $[t(30)=$ $2.104, p<.05]$. Therefore, the absence of overall contingency awareness reported by Dickinson and Brown, on aggregate scores overlooked the fact that over a third of the participants in their experiment were aware of at least three out of four of the contingencies. Furthermore, only that subset of participants who were aware exhibited a significant effect of evaluative conditioning; thus, it appears that Dickinson and Brown's data are consistent with our finding of a concordance between contingency awareness and evaluative conditioning (Experiments 1 and 2A).

As evaluative conditioning using flavor stimuli appears to depend on contingency awareness, the possibility of demand awareness as a source of this evaluative conditioning effect needs to be acknowledged. Participants would be classified as demand aware if they realized that we expected them to rate the flavor previously paired with a sweet taste more positively than the flavor mixed with a bitter taste (see, e.g., Shanks \& St. John, 1994). Demand awareness could account for the finding that only the aware group displayed evaluative conditioning in our study and in that of Dickinson and Brown (2007); participants may have responded on the evaluative test according to what they thought was expected of them, rather than according to their genuine evaluation of the flavors. The results of Experiment 2B suggest that this explanation of our evaluative conditioning effect is unlikely, however. Participants were aware of the contingencies for color CSs, yet there was no evaluative conditioning effect. If contingency awareness drives evaluative conditioning, there is no reason why this should be dependent on the type of CS.

This raises the further issue of why we did not observe color evaluative conditioning. Baeyens, Eelen, et al. (1990) suggest that their failure to obtain color condition-

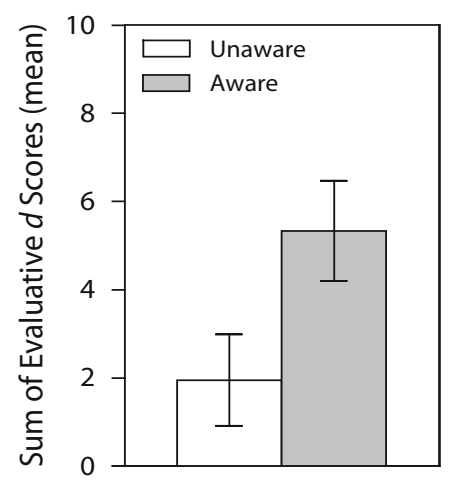

Figure 4. Amount of evaluative conditioning in aware and unaware participants in Dickinson and Brown (2007). Error bars indicate standard errors of the means. 
ing may be because of selective associations in humans' acquisition of food likes and dislikes. This is certainly a possibility. It is also possible that the difference lies in the CS-US contiguity. Flavor CSs are experienced simultaneously with the US tastes as a single stimulus. The color CSs, however, are distinct from the USs and are presented sequentially (the drink is seen, then tasted). It is possible that sequential presentation allows contingency awareness but not evaluative conditioning; that is, contingency awareness is necessary but not sufficient to see evaluative conditioning.

Alternatively, the absence of evaluative conditioning with colors may simply have been a consequence of the instruction to focus on the taste of each sample; perhaps participants ignored the colors. However, this seems unlikely, for two reasons: Firstly, many participants were aware of the color-US contingencies but did not show evaluative conditioning; and secondly, participants in Baeyens, Eelen, et al. (1990), who also failed to show color evaluative conditioning, were informed that the study was on humans' perception of different colors and tastes. It is clear that colors and flavors behave differently when paired with sweet and bitter tastes. The exact nature of this difference remains to be explored.

In summary, the present experiments are the first to demonstrate awareness of contingencies in flavor evaluative conditioning. Moreover, only those participants classified as aware demonstrated evaluative conditioning. The result is compelling, because-despite the simplicity of the design - there was a subgroup of participants who were classified as unaware and who displayed no evaluative conditioning. Significantly, the apparent contradiction between the present study and that of Dickinson and Brown (2007) was resolved by separating aware from unaware participants in their experiment. This reanalysis revealed that only aware participants displayed evaluative conditioning. Therefore, the present study, and that of Dickinson and Brown, demonstrate that evaluative conditioning with flavor stimuli may not be possible when participants are unaware of the CS-US contingencies.

\section{AUTHOR NOTE}

Correspondence concerning this article should be addressed to C. Mitchell, School of Psychology, University of New South Wales, Sydney, NSW 2052, Australia (e-mail: chris.mitchell@unsw.edu.au).

\section{REFERENCES}

Baeyens, F., Crombez, G., Hendrickx, H., \& Eelen, P. (1995). Parameters of human evaluative flavor-flavor conditioning. Learning \& Motivation, 26, 141-160.

Baeyens, F., Crombez, G., Van den Bergh, O., \& Eelen, P. (1988). Once in contact always in contact: Evaluative conditioning is resistant to extinction. Advances in Behaviour Research \& Therapy, 10, 179-199.

Baeyens, F., Eelen, P., \& Van den Bergh, O. (1990). Contingency awareness in evaluative conditioning: A case for unaware affectiveevaluative learning. Cognition \& Emotion, 4, 3-18.

Baeyens, F., Eelen, P., Van den Bergh, O., \& Crombez, G. (1990). Flavor-flavor and color-flavor conditioning in humans. Learning \& Motivation, 21, 434-455.
DAVEY, G. C. L. (1994). Is evaluative conditioning a qualitatively distinct form of classical conditioning? Behaviour Research \& Therapy, 32, 291-299.

De Houwer, J. (2001). Contingency awareness and evaluative conditioning: When will it be enough? Consciousness \& Cognition: An International Journal, 10, 550-558.

De Houwer, J., Thomas, S., \& Baeyens, F. (2001). Association learning of likes and dislikes: A review of 25 years of research on human evaluative conditioning. Psychological Bulletin, 127, 853-869.

Dickinson, A., \& Brown, K. J. (2007). Flavor evaluative conditioning is unaffected by contingency knowledge during training with colorflavor compounds. Learning \& Behavior, 35, 36-42.

Field, A. P. (2000). I like it, but I'm not sure why: Can evaluative conditioning occur without conscious awareness? Consciousness \& Cognition: An International Journal, 9, 13-36.

Field, A. P., \& DaVey, G. C. L. (1999). Reevaluating evaluative conditioning: A nonassociative explanation of conditioning effects in the visual evaluative conditioning paradigm. Journal of Experimental Psychology: Animal Behavior Processes, 25, 211-224.

FulChER, E. P., \& CocKs, R. P. (1997). Dissociative storage systems in human evaluative conditioning. Behaviour Research \& Therapy, 35, 1-10.

Fulcher, E. P., \& Hammerl, M. (2001a). When all is considered: Evaluative learning does not require contingency awareness. Consciousness \& Cognition: An International Journal, 10, 567-573.

Fulcher, E. P., \& Hammerl, M. (2001b). When all is revealed: A dissociation between evaluative learning and contingency awareness. Consciousness \& Cognition: An International Journal, 10, 524-549.

Hammerl, M. (2000). I like it, but only when I'm not sure why: Evaluative conditioning and the awareness issue. Consciousness \& Cognition: An International Journal, 9, 37-40.

Levey, A. B., \& Martin, I. (1975). Classical conditioning of human "evaluative" responses. Behaviour Research \& Therapy, 13, 221226.

Lovibond, P. F., \& Shanks, D. R. (2002). The role of awareness in Pavlovian conditioning: Empirical evidence and theoretical implications. Journal of Experimental Psychology: Animal Behavior Processes, 28, 3-26.

O'BRIEN, R. G., \& KAISER, M. K. (1985). MANOVA method for analyzing repeated measures designs: An extensive primer. Psychological Bulletin, 97, 316-333.

Pleyers, G., Corneille, O., Luminet, O., \& Yzerbyt, V. (2007). Aware and (dis)liking: Item-based analyses reveal that valence acquisition via evaluative conditioning emerges only when there is contingency awareness. Journal of Experimental Psychology: Learning, Memory, \& Cognition, 33, 130-144.

Shanks, D. R., \& ST. John, M. F. (1994). Characteristics of dissociable human learning systems. Behavioral \& Brain Sciences, 17, 367-447.

Shimp, T. A., Stuart, E. W., \& EngLe, R. W. (1991). A program of classical conditioning experiments testing variations in the conditioned stimulus and context. Journal of Consumer Research, 18, 1-12.

Stevenson, R. J., Boakes, R. A., \& Prescott, J. (1998). Changes in odor sweetness resulting from implicit learning of a simultaneous odor-sweetness association: An example of learned synesthesia. Learning \& Motivation, 29, 113-132.

Vansteenwegen, D., Francken, G., Vervliet, B., De Clerce, A., \& Eelen, P. (2006). Resistance to extinction in evaluative conditioning. Journal of Experimental Psychology: Animal Behavior Processes, 32, 71-79.

Walther, E., \& Nagengast, B. (2006). Evaluative conditioning and the awareness issue: Assessing contingency awareness with the fourpicture recognition test. Journal of Experimental Psychology: Animal Behavior Processes, 32, 454-459.

Zellner, D. A., Rozin, P., Aron, M., \& Kulish, C. (1983). Conditioned enhancement of human's liking for flavor by pairing with sweetness. Learning \& Motivation, 14, 338-350.

(Manuscript received March 23, 2007; revision accepted for publication July 25, 2007.) 